Ann. Zootech., I969, 18 (4), 407-4I8.

\title{
RELATION PERMETTANT DE CORRIGER RAPIDEMENT ET AVEC PRÉCISION LA MATIERE SECHE DES ENSILAGES SÉCHÉS A L'ÉTUVE
}

\author{
Nathalie FATIANOFF et Ph. GOUET* \\ Laboratoive de Recherches sur la Conservation et l'Efficacité des Aliments, \\ 16, rue Claude Bernard, 75 - Paris (5e) \\ Institut national de la Recherche agronomique
}

\section{SOMMAIRE}

Les produits volatils (acides gras à courtc chaîne, ammoniaque, alcool) présents en quantités variables dans les ensilages sont toujours partiellement ou totalement perdus au cours du séchage, quelle que soit la méthode utilisée. Ces pertes peuvent atteindre ou dépasser ro $\mathrm{p}$. roo de la matière sèche. Il est donc nécessaire de corriger la matière sèche doséc du poids des substances volatilisées, ce qui implique le dosage de celles-ci dans le produit frais, puis dans le produit sec.

Pour éviter la seconde analyse, des méthodes de correction simplifiée ont été proposées (Dewar et MC DONALD, I96I; SCHOCH, I949). La présente étude a pour but de vérifier, et éventuellement d'ajuster la relation calculée par Sсносн (1949) compte tenu de l'emploi de nouvelles techniques d'analyse et de la présence d'ensilages dont la gamme de taux de matière sèche est plus étendue.

La matière sèche de 59 ensilages est déterminée par dessiccation en étuve à $70^{0}-80^{\circ} \mathrm{C}$ pendant I 6 à 24 heures et corrigée après dosage des acides gras volatils et de l'ammoniaquə dans le produit frais, puis dans la matière sèche.

Ces ensilages sont préparés à partir de différents fourrages avec ou sans traitement. Les taux de matière sèche vont de 18 à $56 \mathrm{p}$. Ioo, et la qualité est très variée.

Les résultats obtenus montrent qu'il existe une relation linéaire et une corrélation élevée $(r=0,94)$ entre le facteur de correction $(\mathrm{Y})$ de la matière sèche et la quantité $(\mathrm{X})$ de composés volatils (acides gras volatils et ammoniaque) dosés sur le produit frais et exprimés en p. Ioo de la matière sèche non corrigée :

$$
\mathrm{Y}=\mathbf{I}, 00+0,007 \mathrm{X}
$$

matière sèche corrigée $=$ matière sèche non corrigée $X \mathrm{Y}$

Dans ces conditions, la matière sèche corrigée peut être obtenue avec une précision égale ou supérieure à I p. Ioo. Cette relation cst très voisine de celle de ScHoch (1949) :

$$
\mathrm{Y}=\mathrm{I}, \mathrm{00}+\mathrm{0}, 008 \mathrm{X} \text {. }
$$

La méthode proposée, aussi précise que celle de l'entrainement de l'eau par le toluène, a en outre l'avantage d'être mieux adaptée aux analyses en série, et d'utiliser des échantillons de dimensions suffisantes pour limiter les erreurs dues à l'hétérogénéité des ensilages.

(I) Adresse actuelle : Laboratoire de microbiologie, C, R. Z, V 63-Theix par Saint-Genès-Champanelle. 


\section{INTRODUCTION}

La détermination de la matière sèche d'un produit consiste en principe à éliminer la totalité de l'eau sans entraîner les substances volatiles initialement présentes ou formées au cours du séchage par la dégradation de composés labiles.

Parmi les techniques applicables aux ensilages, certaines sont peu employées dans les laboratoires en raison de leur manque de précision mais peuvent être utiles lorsqu'une détermination approximative et surtout très rapide est nécessaire: méthode à l'huile (DEXTER, I948), à l'acide sulfurique, au carbure de calcium (PARKS, I94I). Celles qui font appel à la dessiccation sous pression réduite, en particulier la lyophilisation, sont précises et permettent d'éviter les dégradations ou remaniements des constituants les plus labiles (sucres, acides aminés, vitamines). Cependant, dans les études sur ensilages qui comportent souvent un grand nombre d'échantillons de volume important, la seule détermination de routine de la matière sèche nécessiterait un équipement semi-industriel coûteux. De ce fait, les méthodes les plus couramment employées sont la dessiccation à l'étuve à ventilation statique ou forcée et l'entraînement de l'eau par un solvant, tel que le toluène. Quelle que soit la solution retenue, les substances volatiles des ensilages (acides gras volatils, ammoniaque, alcools) sont toujours partiellement perdues au cours du séchage et ces pertes peuvent atteindre ou même dépasser Io p. Ioo de la matière sèche, (FENner et BARnEs, I965; Forbes, I943; IAANIGAN, I96I ; MCDONAID et DEWAR, I960 ; MINSON et LANCASTER, I963; Perkins, I943 ; SCHOCH, I949 ; ToDorow, r967 ; Wilson et al., r964). Pour Colovos et col. (I957), la dessiccation des ensilages à l'étuve entraîne une perte de matières azotées totales de 22 à 37 p. Ioo, et d'énergie brute de 8 à 20 p. Ioo. Cette sous-estimation du taux de matière sèche des ensilages a pour conséquence une surestimation des pertes apparentes dans les bilans de conservation. Pour la même raison, les quantités supposées ingérées par les ruminants se trouvent sous-estimées.

Pour les ensilages, il est donc nécessaire de corriger la matière sèche dosée du poids des substances volatilisées, ce qui implique leur dosage dans le produit avant et après dessiccation. Afin d'éviter la seconde opération, DEwar et McDonal, ont mis au point en I96I une détermination directe de la matière sèche par distillation dans le toluène qui est précise mais trop longue pour être appliquée à des séries. Or, en I959, ScHoch avait déjà proposé une méthode de correction simple, basée sur le fait que la quantité de composés volatils perdus au cours du séchage de l'ensilage à l'étuve est proportionnelle à leur concentration dans le produit frais, quand le résultat est exprimé en p. roo de la matière sèche non corrigée. Il a ainsi établi une relation qui permet de calculer un facteur de correction pour chaque taux de matière sèche d'ensilage. Toutefois, cette relation a été calculée à partir des résultats fournis par des ensilages à faible teneur en matière sèche, après dessiccation à $60^{\circ} \mathrm{C}$ pendant I6 heures puis à I0 $5^{\circ} \mathrm{C}$ pendant 3 heures, et dosage des acides gras volatils par distillation fractionnée selon la méthode de LEEPER (I938). Or, à 1'heure actuelle, il est recommandé, et souvent admis, de déterminer la matière sèche des fourrages à $70^{\circ} \mathrm{C}$ (A. F. Z., I959) ; par ailleurs, les laboratoires de recherches utilisent de plus en plus, pour doser les acides gras volatils, la chromatographie en phase gazeuse plutôt que 
la méthode de LEPPER (I938) employée par Schoch (I949). Enfin, la proportion d'ensilages à haute teneur en matière sèche augmente chaque année. Cette étude se propose de vérifier et éventuellement d'ajuster la relation établie par ScHoch (I949), compte tenu de l'emploi de nouvelles méthodes d'analyses, et de la présence d'ensilages ayant une gamme de taux de matière sèche plus étendue.

\section{PROTOCOLE, EXPÉRIMENTAL,}

\section{Types d'onsilages analysés}

Cinquante-neuf ensilages de luzerne, luzerne-graminée, fétuque, ray-grass ont été analysés. Les traitements subis par ces ensilages se répartissent comme suit :

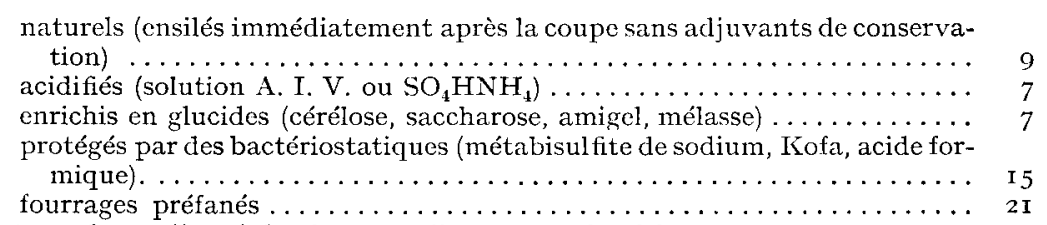

Les taux de matière sèche de ces ensilages vont de $\mathbf{r} 8$ à $56 \mathrm{p}$. 100 .

\section{Détermination de la matière sèche}

Le prélèvement d'ensilage, représentant I à 3 p. Ioo de la quantité totale à échantillonner, est obtenu soit par sondage, soit directement au fur et à mesure du désilage. Après homogénéisation, on prélève deux ou trois échantillons de $300 \mathrm{~g}$ pour les ensilages à teneur en matière sèche élevée (50 p. roo et plus) et de $500 \mathrm{~g}$ pour les plus humides. Les pesées sont effectuées dans des plateaux non perforés, émaillés, de $42 \times 32 \times 5 \mathrm{~cm}$ sur une balance sensible à $0,5 \mathrm{~g}$. Les plateaux sont placés dans une étuve à ventilation forcée réglée à $70^{\circ} \mathrm{C}$, ou dans un four à chauffage par lampes infrarouges. Dans ce cas, la température est maintenue à $80-85^{\circ} \mathrm{C}$ pendant les 2 ou 3 premières heures, puis à $70^{\circ} \mathrm{C}$ pour le reste du temps. Le produit sec est pesé une première fois après 16-20 heures, puis toutes les 2 heures jusqu'à poids constant.

\section{Dosage des composés volatils et correction de la matière sèche}

Cinquante grammes d'ensilage frais ou sec sont mis à macérer dans $45^{\circ} \mathrm{ml}$ d'eau pendant I 7 heures à $0-4^{\circ} \mathrm{C}$. On dose sur le filtrat l'azote ammoniacal selon la méthode de Conway (I96o) et les acides gras volatils (A. G. V.) d'après celle de James et Martin modifiée par ZeLrer (I958). Pour chaque ensilage, ces dosages sont effectués dans le produit frais puis dans le produit séché à l'étuve. La quantité (D) de produits volatilisés au cours du séchage est obtenue par différence ; lorsque $\mathrm{D}$ est exprimé en $\mathrm{p}$. Ioo de la matière sèche non corrigée (M. S. n. c.) la correction de la matière sèche dosée peut être effectuée par l'emploi d'un facteur de correction (Y) :

$$
\begin{gathered}
\mathrm{Y}=\mathrm{I}+\frac{\mathrm{D}}{\mathrm{IOO}} \\
\mathrm{MS} \text { corrigée }=\text { MS n. c. } \times \mathrm{Y}
\end{gathered}
$$

\section{RÉSULTATS}

\section{I. Échantillonnage et séchage}

La perte de poids observée après $16-20$ heures de séchage s'accentue rarement lorsque les échantillons sont remis à l'étuve pendant 2 autres heures. Toutefois, si celle-ci est entièrement remplie d'échantillons très humides (I5-20 p. roo M. S.) en 
début de séchage, on peut enregistrer au cours de cette phase complémentaire des pertes de poids de 0,5 à I p. I00, ce qui oblige à prolonger la dessiccation durant deux nouvelles heures. I a constance du poids est toujours atteinte dans un délai de I 6 à 24 heures.

Les écarts constatés entre les M. S. n. c. déterminées en double sur un même échantillon proviennent principalement de l'hétérogénéité du fourrage, même haché fin $(\mathrm{I}-2 \mathrm{~cm}): 75 \mathrm{p}$. Ioo des échantillons examinés ont un écart, par rapport à la moyenne, inférieur à I p. Ioo et 93 p. Ioo inférieur à 2 p. Ioo. Des résultats identiques ont par ailleurs été enregistrés pour les fourrages verts à la mise en silo.

Quant aux écarts observés pour des échantillons semblables entre 1'étuve à ventilation et celle à chauffage infrarouge, ils sont identiques à ceux que 1'on enregistre entre deux échantillons semblables séchés dans la même étuve.

\section{Pertes de composés volatils et correction de la matière sèche}

Les ensilages frais analysés sont de qualité très variable puisque leur teneur en substances volatiles (A. G. V. $+\mathrm{NH}_{3}$ ) varie entre 0,4 et 9,8 p. Ioo de la M. S. n. c. (tab1. I). Les concentrations extrêmes en acide acétique et en ammoniaque, présents dans tous les ensilages, varient respectivement de 0,2 à 7 p. Ioo et de 0,2 à $I, 6$ p. Ioo de la M. S. n. c. Deux tiers des échantillons contiennent de l'acide propionique (teneur maximum : I, I p. Ioo M. S. n. c.) et de l'acide butyrique (teneur maximum : 4,4 p. Ioo M. S. n. c.). Deux échantillons sur les 59 analysés contiennent de l'acide valérianique (o,I et 0,2 p. Ioo M.S. n. c.).

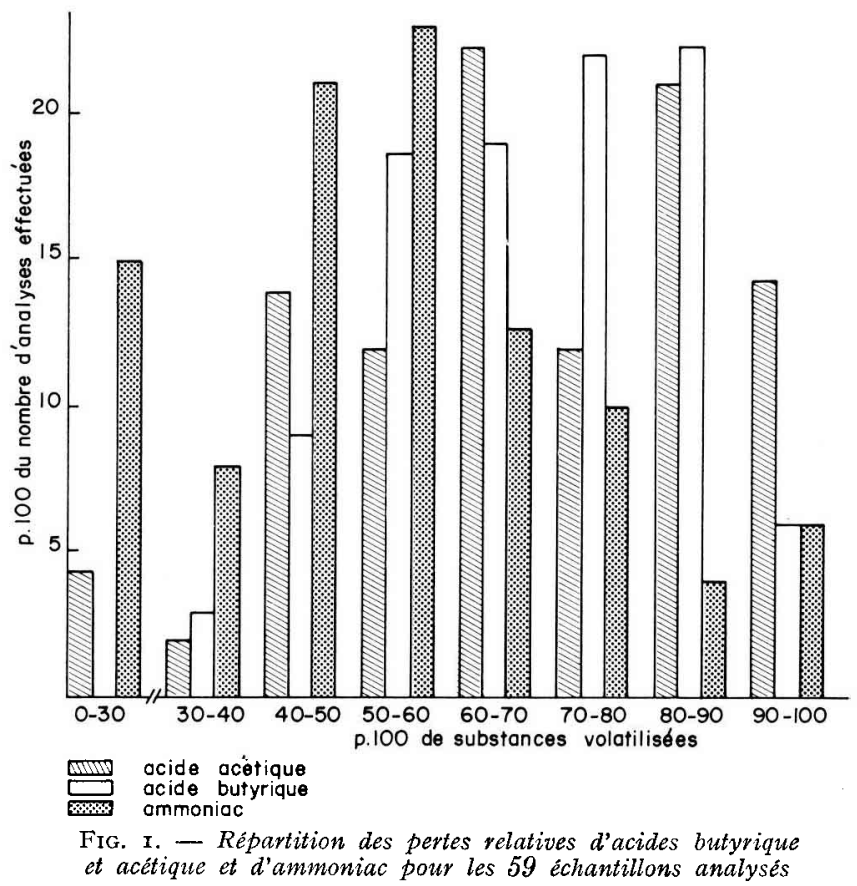


Les proportions de substances volatilisées lors du séchage à l'étuve sont extrêmement variables (fig. I) et vont de Io à $98 \mathrm{p}$. Ioo pour l'acide acétique, 30 à $92 \mathrm{p}$. Ioo pour l'acide butyrique et o à 92 p. Ioo pour l'ammoniaque. En moyenne, elles sont respectivement de 68 p. I00, 69 p. Ioo et 54 p. 100. Aucune relation n'a pu être établie entre les proportions de substances volatilisées et leur concentration dans l'ensilage.

Rapportées à la M. S. n. c., les pertes globales de composés volatils (A.G.V.+NH $\mathrm{NH}_{3}$ ) varient entre 0,2 et $6,9 \mathrm{p}$. Ioo. Il existe une liaison linéaire et une corrélation élevée $(r=0,94)$ entre le facteur de correction $(Y)$ de la matière sèche et la quantité $(X)$ de composés volatils dosés sur l'ensilage frais et exprimée en p. Ioo de la M. S. n. c. (fig. 2) :

$$
\mathrm{Y}=\mathrm{I}, 00+0,007 \mathrm{X} \text {. }
$$

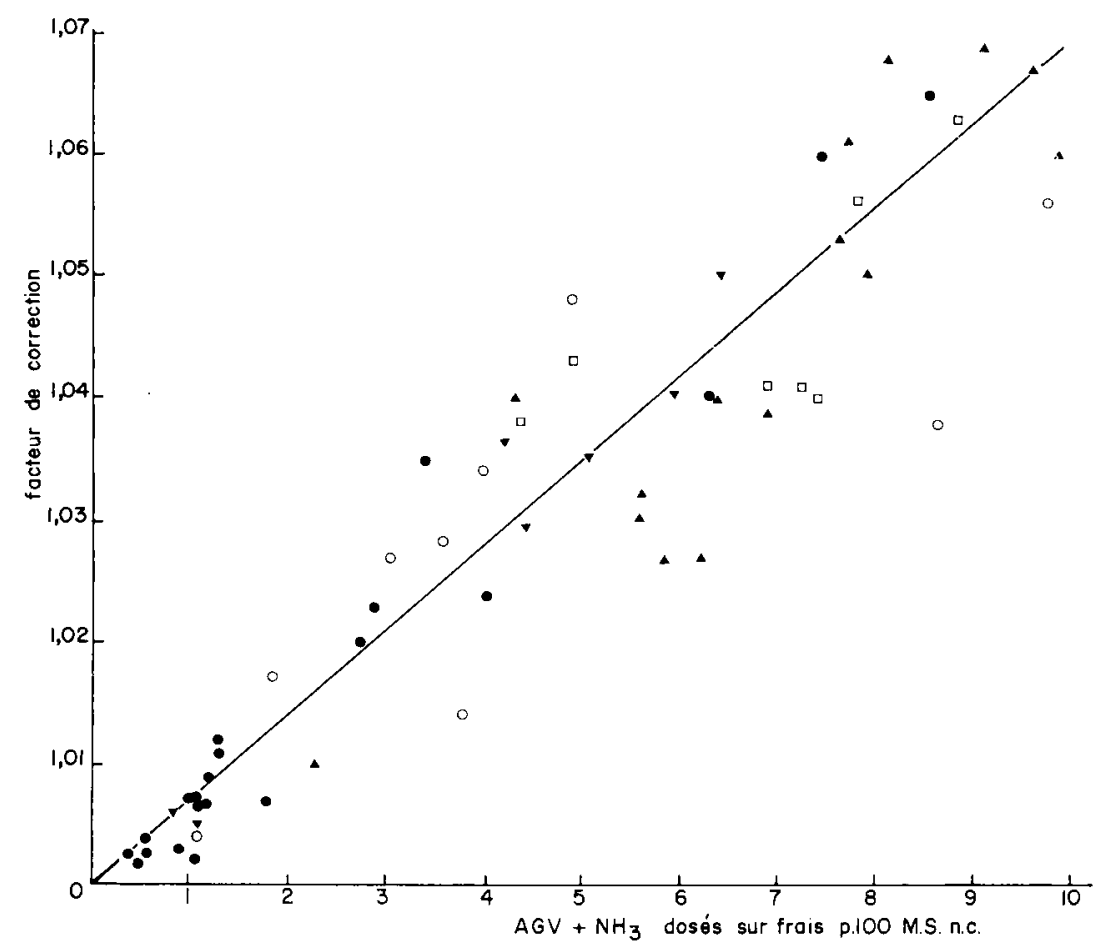

FIG. 2. -- Relation entre la teneur de l'ensilage en composés volatils dosés sur frais et le facteur de correction de la $M$.S.

$y=\mathrm{I}, 00+0,007 x ; n=59 ; r=0,94$

Ensilages : 0 naturels

- avec addition de bactériostatiques

$\square$ enrichis en sucres

$\checkmark$ acidifiés

- préfanés

L'écart entre les facteurs de correction calculés à partir des résultats d'analyse sur frais et sur sec $(\mathrm{Y} a)$ et par l'emploi de cette relation $(\mathrm{Y} r)$ est inférieur ou égal à I p. Ioo dans 86 p. Ioo des analyses, I,5 p. Ioo dans 93 p. Ioo et 2 p. Ioo dans 98 p. roo (tabl. I).

On remarquera enfin que l'importance de la correction est liée à la qualité de 


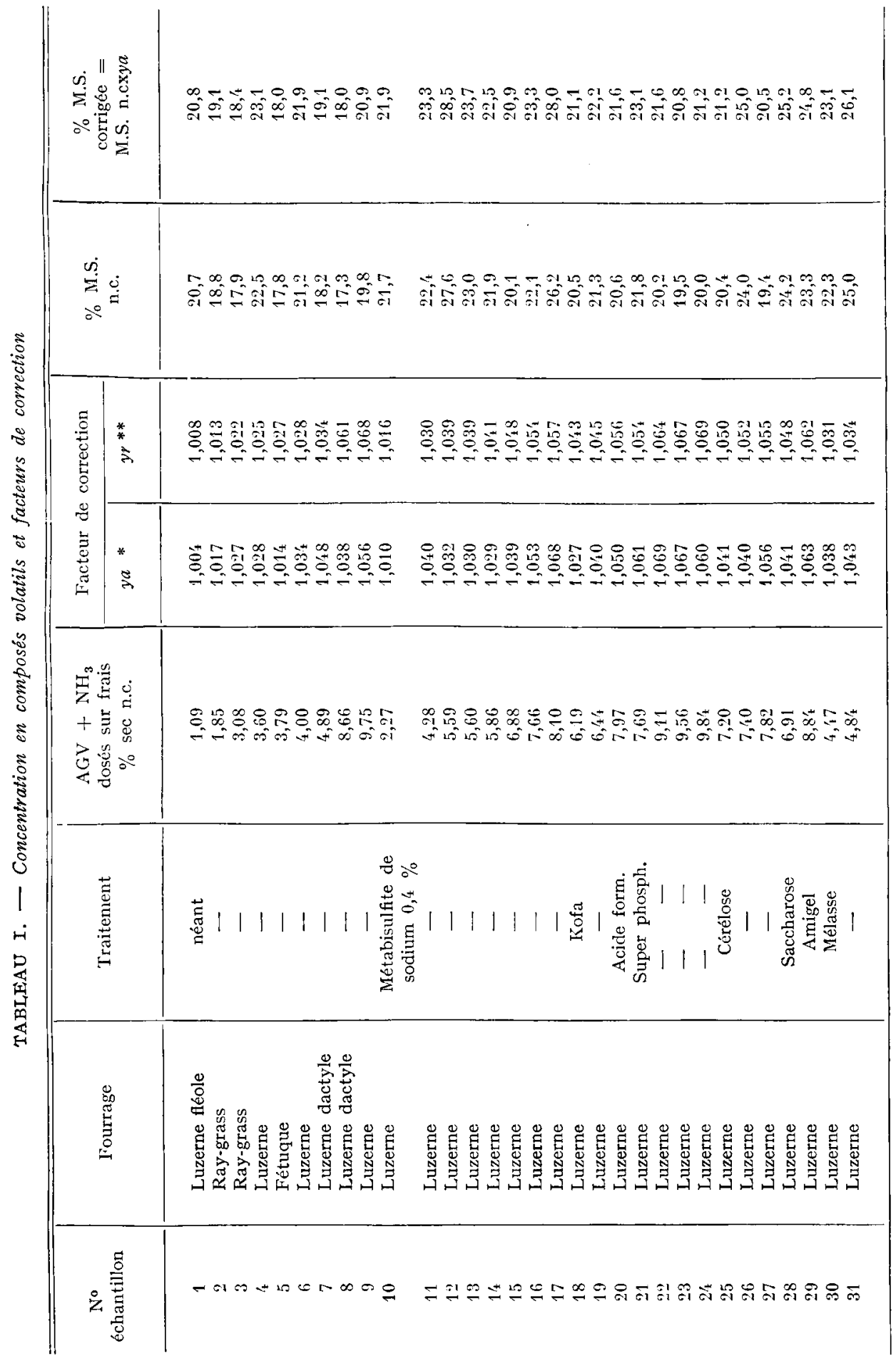


CORRECTION DE LA MATIÈRE SÈCHE DES ENSILAGES

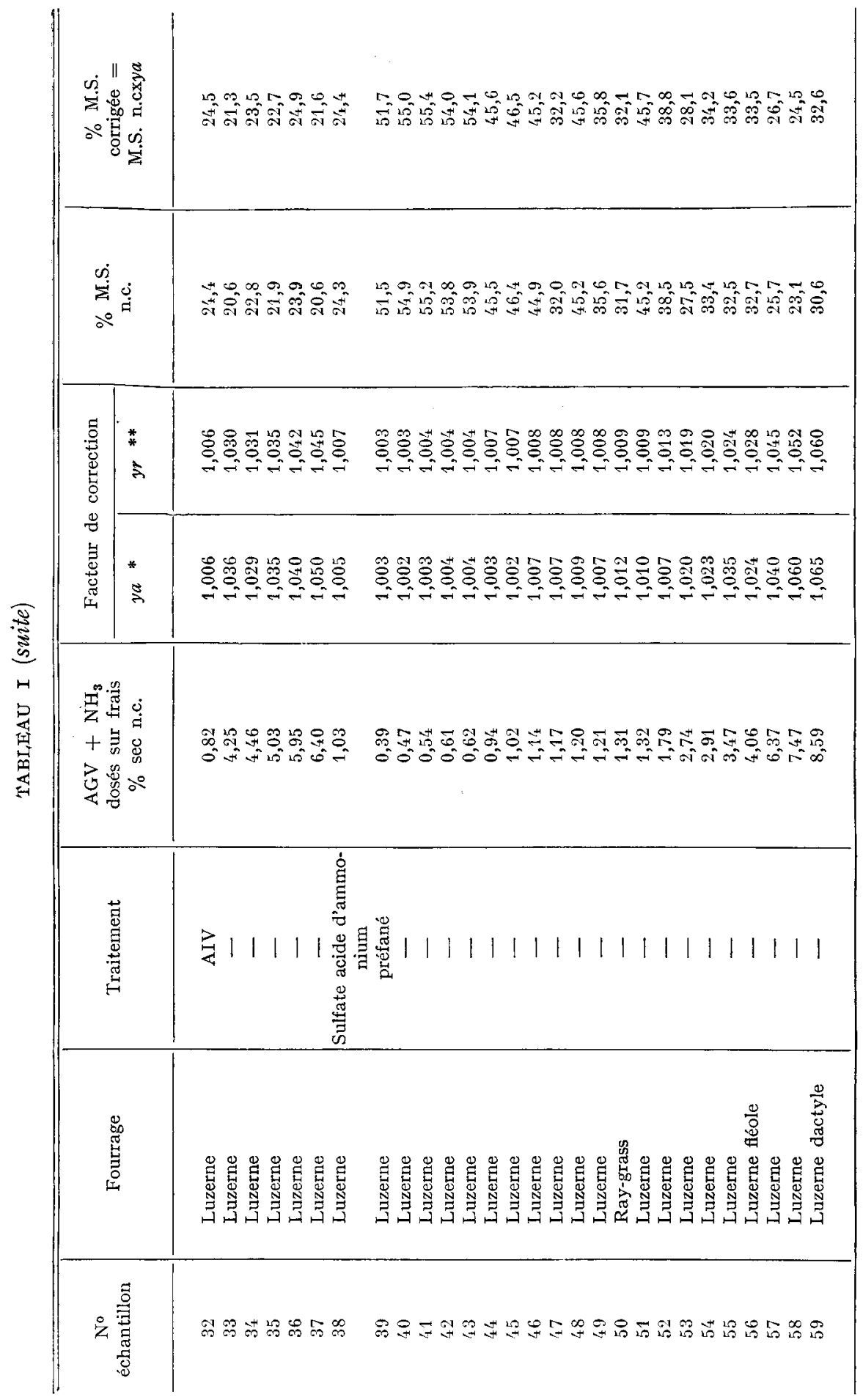


l'ensilage. Parmi les traitements, seule l'élévation du taux de matière sèche réduit fortement l'activité fermentaire lorsque les ensilages sont conservés en atmosphère anaérobie ; la correction devient alors très faible (ensilages 39 à 46 ; tabl. I).

\section{DISCUSSION}

Nos résultats, ceux de Schoch (I949), MCDonald et DEWar (I960), Watson et FERGUSON (I937) (tabl. 2), obtenus avec des méthodes de séchage et d'analyse différentes, montrent une grande variabilité des pertes relatives d'acides gras volatils et d'ammoniaque. Cette variabilité est essentiellement liée au $\mathrm{pH}$ et au pouvoir tampon de 1'ensilage qui déterminent les proportions d'A. G. V. et $\mathrm{NH}_{3}$ libres ou sous forme de sels plus ou moins sujets à la volatilisation (SCHOCH, I949; WoODMAN, I925).

Le séchage à $105^{\circ} \mathrm{C}$ au lieu de $70^{\circ} \mathrm{C}$ tend toutefois à augmenter les pertes relatives des A. G. V. de I5 à 25 p. Ioo en moyenne (tab1. 2). C'est probablement pour cette raison que le facteur de correction calculé par $\mathrm{ScHocH}$ (I949) est légèrement supérieur au nôtre $: \mathrm{Y}=\mathrm{I}, 00+0,008 \mathrm{X}$. L'écart entre les deux relations est toutefois très faible en regard des différences de température, de durée de séchage et des méthodes de dosage employées. La confirmation de ce fait peut être trouvée en appliquant la relation proposée ( $\mathrm{Y}=\mathrm{I}+0,007 \mathrm{X}$ ) ainsi que celle de $\mathrm{SCHOCH}$ ( $\mathrm{I} 949$ ) aux résultats de McDonal, (Edinburgh School of Agriculture, I960) obtenus par le double dosage de douze échantillons séchés à $100^{\circ} \mathrm{C}$. Les moyennes des résultats obtenus par les trois méthodes sont respectivement 17,52 , I7,56 et I7,6I. On aboutit donc à des valeurs très voisines, les écarts moyens étant respectivement de $-0,5$ et $-0,3$ p. Ioo.

On peut donc admettre que la température, dans les limites indiquées $\left(70-105^{\circ} \mathrm{C}\right)$, et pour des durées de séchage n'excédant pas 24 heures, influence très peu le facteur de correction de la M. S. Mais la dessiccation à 100-10 $5^{\circ} \mathrm{C}$, surtout au-delà de 24 heures risque de provoquer des pertes de substances labiles non identifiées, qui sont quasiment nulles au-dessous de $80^{\circ} \mathrm{C}$ (BARNETT, I954; GREFNHILI, I960 ; LFROY, I954; Minson et Lancaster, I963; SaI,O, I965).

Alors que la relation de $\mathrm{ScHOCH}$ (1949) paraît totalement ignorée, la méthode de distillation en présence de toluène est, par contre, préconisée par de nombrenx auteurs anglo-saxons et allemands (DEWAR et MCDONALD I96I; FENNER I967; FenNer et Barnes ig65; Minson et Lancaster ig63; Spiliane et Wilson i967; ROHR et FENNER I967; WiLsON et col. I964).

Cette technique, complétée par la correction de DEWAR et McDonald (I96I) aboutit à des résultats identiques (écart inférieur à I p. IOO) à ceux de la dessiccation en étuve à $100^{\circ} \mathrm{C}$ corrigés par dosage des composés volatils sur les matières fraîches et sèches, soit, en définitive, une précision comparable à celle que l'on peut obtenir par l'emploi d'un facteur de correction. Elle nécessite en outre un appareillage encombrant si le nombre d'échantillons est important, la manipulation de solvants toxiques. et une surveillance de 5 à 7 heures. Enfin, elle n'est vraiment adaptée qu'à des ensilages humides (2I p. IOo M. S. maximum) et le poids réduit de l'échantillon risque d'augmenter l'erreur due à l'échantillonnage (DEWAR et McDonal,d I96I ; SPILLANE: et Wirson I967). 


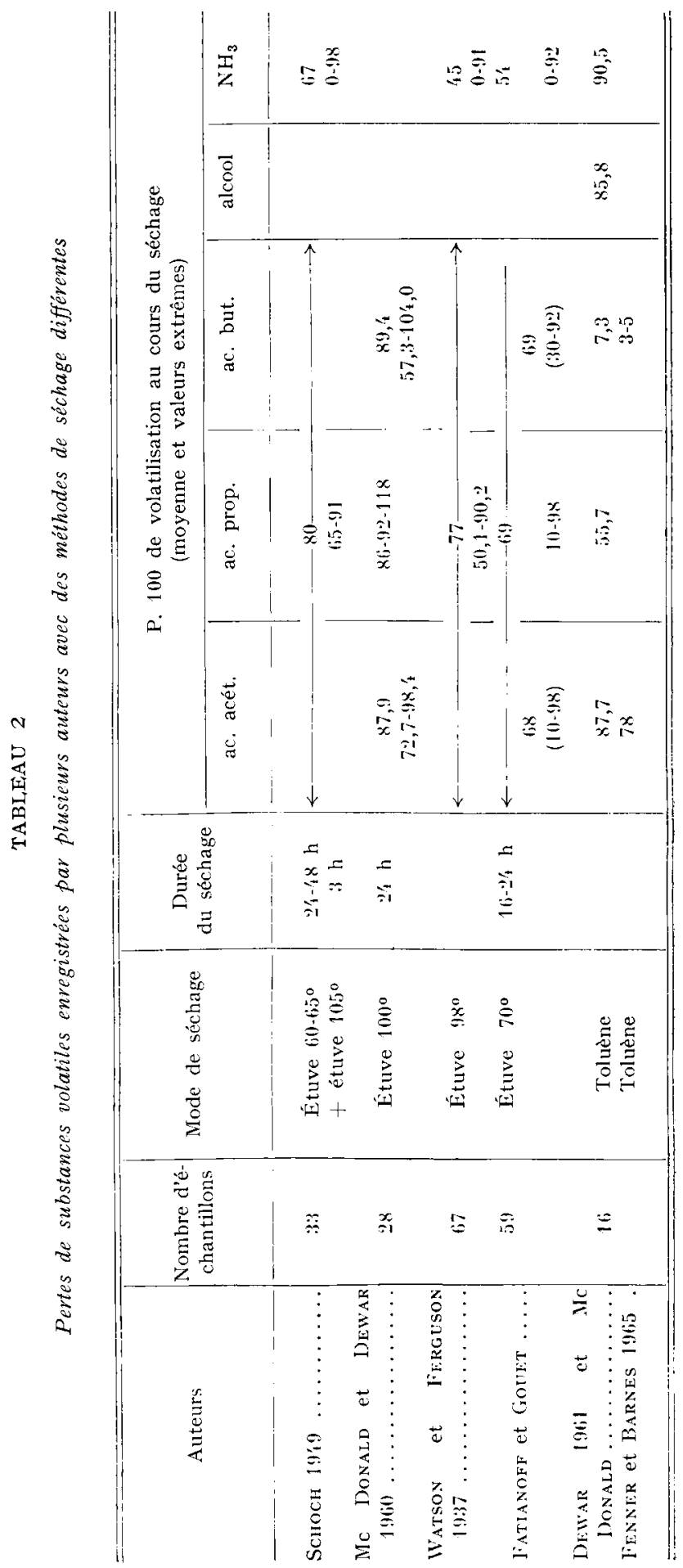


L'acide formique, les acides volatils aut-delà de $\mathrm{C}_{4}$ et les amines volatiles parfois présentes dans les ensilages, ne justifient pas une analyse en vue de la correction de la M. S. car leur concentration est toujours très faible et ne risque pas d'entraîner une erreur sensible (Jackson, I964; Mac Pherson I966; Voss, I966).

Parmi les substances volatiles dont la détermination n'est pas courante, l'alcool (volatil à Ioo $\mathrm{p}$. IOO) peut constituer une fraction non négligeable de la matière sèche. Cela n'est pas le cas pour les ensilages de graminées et de légumineuses où l'alcool est absent ou à l'état de traces (McDonald et DEWAR, I960). Par contre, nous avons observé (résultats non publiés) des concentrations atteignant 4 p. Ioo de la M. S. dans des ensilages de maïs fourrage très humide ( $5^{-I}$ 7 p. Ioo M. S.), I,5 p. Ioo dans du mais grain, 3 p. Ioo dans des feuilles et collets de betterave. Sa présence est signalée aussi dans les pulpes ensilées, les pommes et mares de pomme (LEROY, $Z_{\text {ELTER, CHAR- }}$ LEET-LÉRY I957).

\section{CONCLUSION}

Il est bien établi que le résultat du dosage de la matière sèche d'un fourrage ensilé varie légèrement suivant la température et la durée du séchage. Le choix de la valeur de ces deux paramètres ne repose pas sur des comparaisons systématiques et précises et reste, de ce fait, assez conventionnel. Toutefois nos résultats ainsi que ceux d'expériences partielles réalisées par divers auteurs (BARNETT, I954; GREENHILI, I960 ; LEROY, I954; Minson et LANCASTER, I963 ; SALO, I965) convergent et permettent de conclure qu'une température de $70^{\circ} \mathrm{C}$ environ est celle qui dégrade ou volatilise le moins les composants labiles des fourrages ensilés ou non. La đurée de séchage, déterminée par la constance du poids qui peut être atteinte après I6-20 heures, quel que soit le type d'étuve utilisé, ne doit pas dépasser 24 heures. Si ces conditions sont respectées, l'application de la relation : $\mathrm{Y}=\mathrm{I}, 00+0,007 \mathrm{X}$ au taux de $\mathrm{M}$. $\mathrm{S}$. dosé à l'étuve permet de corriger avec une précision égale ou supérieure à I $\mathrm{p}$. Ioo l'erreur due à la volatilisation des A. G. V. et de l'ammoniaque au cours du séchage. Par contre, si la température de séchage est de $I{ }^{\circ}{ }^{\circ} \mathrm{C}$, il sera préférable d'utiliser la relation de $\mathrm{ScHOCH}$ (I949) : $\mathrm{Y}=\mathrm{I}, \mathrm{OO}+0,008 \mathrm{X}$.

Cette méthode, aussi précise mais moins laborieuse que la distillation en présence de toluène, a l'avantage d'être mieux adaptée aux analyses de série. Elle reste valable dans le cas des ensilages riches en alcool à condition toutefois de doser ce composé sur le produit frais pour apporter la correction appropriée.

Reçu pour publication en novembre 1969.

\section{SUMMARY}

\section{REI,ATION PERMIT'TING TO CORRECT RAPIDLY AND WITH ACCURACY THE DRY MATTER OF OVEN DRIED SILAGES}

In order to take into account volatile losses during oven dried silage, a simple correction procedure is described for an accurate dry matter (D. M.) determination.

59 silage samples were submitted to D. M. determination by oven drying during $16-24 \mathrm{~h}$ at $70-80^{\circ}$ C. Volatile fatty acids (V. F. A.) and ammonia were determined on fresh and dried silage. From these data and for each sample, a correction factor (Y) was calculated and D. M. was corrected (corrected D. M. $\times$ oven D. $\mathrm{M}=\mathrm{Y}$ ). 
Either untreated or treated silages (wilted, acidified, sugar or bacteriostatics addition) have been prepared from different forages (alfalfa, ray grass, alfalfa-grass, mixtures). A large D. M. and silage quality range was obtained.

The data were fitted to the linear regression of correction factor $(Y)$ on total volatile compounds (V. F. A. and ammonia) determined on fresh silage and expressed as per cent oven D. M. The following relation was calculated : $\mathrm{Y}=\mathrm{I} .00+0.007 \mathrm{X} . r=0.94$.

The accuracy and conditions in which this relation can be used are discussed. The results are compared to other simple correction procedures available in the litterature (DEwAR and McDoNALD, I96r. SCHOCH, I 949.). The relation proposed is in good agreement with the one established by Sсносн, (1949) in the same way, but with different analytical procedures and for a not so large ensilage D. M. range. The difference is probably due to the increased V.F.A. losses at the higher drying tempcrature ( $\mathrm{IO}^{\circ} \mathrm{C}$ ) used by this author. The accuracy of this method is similar to the toluene distillation procedure completed with the Foremann titration (DEwar and McDonald, I96I).

Moreover, it is less tedious, best fitted for routine purposes and it allows to work on larger silage quantities, thus to reduce sampling errors.

\section{RÉFÉRENCES BIBLIOGRAPHIQUES}

\section{A. F. Z. Commission d'Alimentation. Sćance du 2 décembre 1959.}

Barnett A. J. G., I954. Silage Fermentation. Butherworths Sci. Publ., London.

Colovos N. F., Keener H. A., Davis H. A., 1957. Errors in drying silage and feces for protein and energy determinations. Improved procedures. J. Dairy Sci, 40, 173-179.

Conway E. J., I960. Microdiffusion analysis and volumetric error. Crossby. London.

Dewar W. A., McDonald P., r96r. Determination of dry matter in silage by distillation with toluene. J. Sici. Fd. Agric., 12, 790-795.

DeXter S. T., I948. Michigan Quart. Bull. November.

Edinburgh school of agriculture, ig6o. Technical, IBull., 24.

Fatianofr Nathalie, (résultats non publiés).

Fenner H., I967. Neuere Methoden Zur Erfassung der wertbestimmenden Bestandteile in Silage. Die Naturwissenschaftlichen Grundlagen der Silierung. Int. Symp. Rostock., 26-28 sept. I966, 221-228.

Fenner H., Barnes H. D., r965. Improved method for determining dry matter in silage. J. Dairy Sci., 48, I324-1 328 .

Forbes E. B., 1943. Pa. Agric. Exp. Sta. Bull., 452.

Greenhill W. L., I960. Determination of the dry weight of herbage by drying methods. J. British. Grassl. Soc., 15, 48-54.

Jackson R. 13., 1964. Volatile bases in raygrass silage. J. Sci. Fd. Agric., 15, 308-312.

Lanigan G. W., r96r. Studies on ensilage. I. Comparative study of molasses and sodium metabisulfite as aids to the conservation of lucerne. Aust. J. Agric. Res., 12, 1023-1038.

LEPPER W., 1938. Zeitschrift für Tievernähr. und Futtermit., 1, 187.

Leroy R., I954. Une méthode correcte de dosage de l'eau. Chimie Anal., 36, 294-3or.

Leroy A. M., Zelter S. Z., Charlet-Léry G., i957. Recherches sur la conservation par ensilage et l'utilisation des poinmes à cidre comme aliment du bétail. Ann. Zootech., 1, I-r.9.

McDonald P., Dewar W. A., 1960. Determination of dry matter and volatiles in silages. J. Sci. Fd A gric., 11, 566-570.

Mac Pherson H. T., Vlolante P., I966. Ornithine, putrescine and cadaverine in grass silage. $J$. Sci. Fd. A gric., 17, 124 -127.

Minson D. J., LANCASTER R. J., Ig63. The effect of oven temperature on the error in estimatirg the dry matter content of silage. N. Z.J.Agric. Res., 6, I40.I46.

PARKS R. Q., I94I. A rapid and simple method for determining moisture in forages and grain. J.Amer Soc. Agron., 33, 325-335. Cité par Parks R. Q., 1941. J. Amer. Soc. Agron., 33, 325-335.

Perisins A. E., 1943. Dry matter determination in green plant material and silage. J. Dairy Sci., 26,. 545-55I.

Rohr K., FENNER H., I967. Der Gehalt einiger Silageproben an wasserreicher Substanz und anderen wertbestimmenden Bestandteilen. Die Naturwissenschaftlichen Grundlagen der Silierung. Int. Symp. Rostock, 26-28 sept. 1966., 299-239.

Salo M. L., 1965 . On the breakdown of sugars during the drying of plant samples and their subsequent dry storage. J. Sci. Agric. Soc. Finland., 37, 186-194.

Annales de Zootechnie. - r 960 . 
Sсносн W., 1949. Die bei der Trocknung von Silageproben im Trockenschrank auftretenden Verluste an flüchtigen Saüren und Basen. Mitteilungen Aus dem Gebiete Der Lebensmitteluntersuchung und Hygiene., 40, I70-189.

SpIllane T. A., Wilson R. I., 1967. Moisture in silage by oven drying and toluene distillation. Anim. Husb. Dairying div. Anim. Sci. Div., I35-I36.

Todorow N. A., I967. Über Methoden zur Bestimmung der Verluste beim Silieren. Die Naturwissenschaftlichen Gründlagen der Silïerung. Int. Symp. Rostock., 26-28 sept. 1966, 265-27o.

Voss N., I966. U̇ber die Amin. und Ammoniakbildung im Gärfutter. Das wirtschafteigene Futter., 2, I6I, I7I.

Watson S. J., Ferguson W. S., 1937. The chemical composition of grass silage. J. Agric. Sci., 27, I-42.

Wilson R. F., Tilley S. M. A., Steemers M. A. Th., I964. Comparaison of oven drying and toluene distillation in the determination of dry matter content of silage. J. Sci. Fd. Agric., 15, I97-200.

Woodman H. Z., 1925. Critical examination of the methods employed in silage analysis with observations on some chemical characteristics of sour silage. J. Agric. Sci., 15, 343-357.

ZELTER S. Z., Leroy F., 1958. Azote uréique et activité bactérienne in vitro au niveau du rumen. Ann. Zootech., 3, 173-183. 